\title{
Adsorption of Cesium and Strontium Ions by Alkali-Treated Rice Hull Charcoal
}

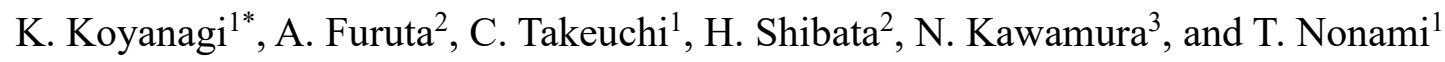 \\ 1 Mechanical and Systems Engineering School of Technology, Chukyo University \\ 2 Graduate School of Information Science, Chukyo University Graduate School \\ 3 Institute for Advanced Studies in Artificial Intelligence, Chukyo University \\ *Corresponding author: k.katsu9222@i.softbank.jp
}

This paper has investigated the influence of alkali treatment on the acidic functional groups of rice hull charcoal and investigated the relationship between its cesium and strontium adsorption ability. In the alkali treatment, rice hull charcoal carbonized from 400 to $1000{ }^{\circ} \mathrm{C}$ was immersed in a basic aqueous solution. The $\mathrm{pH}$ of the rice hull charcoal increased after alkali treatment. Furthermore, the alkali treatment of rice hull charcoal carbonized at 400 and $600{ }^{\circ} \mathrm{C}$ increased the adsorption of cesium and strontium. In the FTIR results, the $\mathrm{OH}$ band of alkali-treated rice hull charcoal was stronger than that of the untreated rice hull charcoal. The mesopore volume of alkali-treated rice hull charcoal increased. The alkali-treated rice hull charcoal showed an increased $\mathrm{pH}$, increased negative charge, and cleaved lactone rings contained compared to the untreated sample. In addition, the valence of acidic functional groups increased, and the adsorption ability of cesium and strontium improved.

Key words: Rice hull, Carbonization, Cesium, Strontium, Acidic functional group, Alkali-treated

\section{INTRODUCTION}

Radioactive substances such as cesium and strontium are released by nuclear accidents and contaminate the atmosphere, soil, and water for a long time. Reducing contamination by radioactive substances reduces their damaging effects to human life. Currently, zeolite is used to adsorb radioactive substances from water and soil. However, the used adsorbent must be securely stored ${ }^{1)}$. Therefore, there is demand for a radioactive adsorbent material to replace zeolite that can be disposed of more easily.

Two million tons of rice hulls are produced in Japan every year ${ }^{2)}$. The production of rice hull charcoal is an effective use of the chaff and has many applications, such as deodorization, water purification, and soil conditioning. There are reports of the adsorption of aldehyde gases using rice hull charcoal ${ }^{3)}$. Furthermore, the adsorption ability, such as the type of substance adsorbed and the adsorption amount, can be adjusted by changing the carbonizing conditions.

Here, we report that rice hull charcoal can adsorb cesium and strontium. Previously, Kobayashi et al. reported that the cesium adsorption of charcoal and bamboo charcoal is affected by the acidic functional groups, such as carboxylic acids and phenolic hydroxyl groups, on the surface of the charcoal ${ }^{4), 5)}$. However, the adsorption capacity of cesium and strontium of rice hull charcoal is inferior to that of zeolite.

Despite this, by treating charcoal in an oxidizing atmosphere at about $400{ }^{\circ} \mathrm{C}$, the carbon component can be removed. Furthermore, the boiling points of cesium and strontium are 671 and $1382{ }^{\circ} \mathrm{C}$, respectively. Therefore, it is possible to burn the rice hull charcoal while leaving these metals as a residue. Therefore, after use in the decontamination treatment, the volume of rice hull charcoal can be greatly reduced. Such volume reduction is difficult with zeolite because it is an inorganic substance. Thus, if the adsorption ability of rice hull charcoal can be improved, it could be used as a substitute for zeolite in part or completely for the adsorption of radioactive material.

It has been reported that rice straw or bamboo charred by helium circulation at $800{ }^{\circ} \mathrm{C}$ for $1 \mathrm{~h}$ and subsequently immersed in aqueous sodium hydroxide shows an increased specific surface area ${ }^{6}$. However, the relationship between the change in the acidic functional groups present on the surface of rice hull charcoal and the adsorption ability of cesium and strontium has not been investigated.

Therefore, in this study, we investigated the relationship between the change in the acidic functional groups of rice hull charcoal by alkali treatment in aqueous base solution and the adsorption ability of cesium and strontium. That is, rice hull charcoal was immersed in sodium hydroxide solution and the acidic functional groups present on the surface of rice hull charcoal were evaluated by Fourier transform (FT) IR measurements. Furthermore, the adsorption ability of rice hull charcoal with cesium and strontium was investigated and the relationship between adsorption and the acidic functional groups was investigated.

\section{EXPERIMENTAL}

2.1 Preparation of rice hull charcoal

First, $10 \mathrm{~g}$ of rice hulls (Aichinohomare, Aichi prefecture 2018) were placed in an alumina crucible and covered. The crucible was heated at $18{ }^{\circ} \mathrm{C} / \mathrm{min}$ in a separator-type desk 
electric furnace (Asahi Rika Seisakusho Co., Ltd.) and carbonized by holding for $1 \mathrm{~h}$ after reaching the set temperature. The set temperatures were $400,600,800$, and $1000{ }^{\circ} \mathrm{C}$. After carbonization, the crucible was allowed to cool in the furnace.

The alkali treatment of rice hull charcoal was carried out as follows: $3.00 \mathrm{~g}$ of rice hull charcoal was added to $30 \mathrm{~mL}$ of aqueous $0.1 \mathrm{~mol} / \mathrm{L} \mathrm{NaOH}(\mathrm{pH} 12.5)$ or $0.10 \mathrm{~mol} / \mathrm{L}$ aqueous $\mathrm{NaHCO}_{3}(\mathrm{pH} 8.7)$ and shaken at $25^{\circ} \mathrm{C}$ for 5 days. After that, the rice hull charcoal was washed with distilled water, and this was repeated until the $\mathrm{pH}$ of the washed aqueous solution became 9. After that, it was naturally cooled.

\subsection{Evaluation}

The $\mathrm{pH}$ was measured in accordance with JIS K 1474 . That is, $100 \mathrm{~mL}$ of water was added to $1.00 \mathrm{~g}$ of the powder sample, and the mixture was gently heated for $5 \mathrm{~min}$ so that boiling continued. On cooling to room temperature, water was added to make the solution volume up to $100 \mathrm{~mL}$. Then, the solution was stirred thoroughly, and the $\mathrm{pH}$ of the rice hull charcoal aqueous solution was measured using a $\mathrm{pH}$ meter (SevenMulti, Mettler Toledo).

The morphology of the rice hull charcoal produced was observed by scanning electron microscopy (SEM, S-2600N, Hitachi High-Technologies).

The specific surface area was measured using the Brunauer-Emmett-Teller (BET) one-point method with flowing nitrogen gas and helium gas and a specific surface area measuring apparatus (Monosorb, Yuasa Ionics Co., Ltd.).

The pore distribution was measured from the amount of adsorbed nitrogen at liquid nitrogen temperature at each relative pressure using a pore distribution measuring apparatus (Autosorb-1, Quantachrome). The measurement was carried out after drying the sample at $117^{\circ} \mathrm{C}$ for $3 \mathrm{~h}$, obtaining the weight, and degassing under reduced pressure at $120^{\circ} \mathrm{C}$

The FTIR spectra of the rice hull charcoal were measured using an FT-IR-680 Plus (JASCO).

The Boehm method was used to measure the total amount of acidic functional groups present on the surface of the rice hull charcoal sample. Rice hull charcoal $(0.10 \mathrm{~g})$ and $300 \mathrm{~mL}$ of a $0.10 \mathrm{~mol} / \mathrm{L} \mathrm{NaOH}$ aqueous solution were added to an Erlenmeyer flask and shaken at $25^{\circ} \mathrm{C}$ for 5 days After filtration of the mixture, the filtrate was back-titrated with a $0.10 \mathrm{~mol} / \mathrm{L} \mathrm{HCl}$ aqueous solution, and the amount of acidic functional groups was determined from the volume of $\mathrm{HCl}$ consumed.

\subsection{Cesium/strontium adsorption experiments}

An aqueous solution of cesium chloride and an aqueous solution of strontium chloride of $100 \mathrm{~mL}$ were prepared so that the concentration of the metallic element was $100 \mathrm{mg} / \mathrm{L}$. To this, $1.00 \mathrm{~g}$ of rice hull charcoal crushed to $250 \mu \mathrm{m}$ or less was added and stirred for $1 \mathrm{~h}$. Thereafter, the solution was filtered through a filter, and the concentrations of metals in the filtrate were measured using an atomic absorption photometer (Z-2300, Hitachi HighTechnologies Corporation). For comparison, the same evaluation was carried out using $1.00 \mathrm{~g}$ of natural zeolite (Nitto Zeolite No. 5, Nitto Powder Industry Co., Ltd.).

\subsection{Cesium/strontium desorption experiments}

Rice hull charcoal carbonized at $400{ }^{\circ} \mathrm{C}$ was dried, and $1.00 \mathrm{~g}$ of the charcoal was added to $100 \mathrm{~mL}$ of distilled water and stirred for $1 \mathrm{~h}$. Thereafter, the solution was filtered through a filter, and the concentrations of the metals in the filtrate were measured using the aforementioned atomic absorption photometer.

\section{RESULTS AND DISCUSSION}

$3.1 \mathrm{pH}$ and specific surface area measurement

Table I lists the $\mathrm{pH}$ of boiling water containing the rice hull charcoal. When the rice hull charcoal treated with alkali was added, the $\mathrm{pH}$ of the boiling water rose. Thus, the $\mathrm{pH}$ of the rice hull charcoal increased after alkaline treatment.

Table I pH and specific surface area of aqueous solution of rice hull charcoal at $400{ }^{\circ} \mathrm{C}$ before and after alkali treatment.

\begin{tabular}{lll}
\hline Sample Name & $\begin{array}{l}\text { Specific Surface } \\
\text { Area } / \mathrm{m}^{2} / \mathrm{g}\end{array}$ & $\mathrm{pH}$ \\
\hline Untreated & 4.78 & 6.59 \\
$0.10 \mathrm{~mol} / \mathrm{L}, \mathrm{NaOH}, 5$ days & 2.78 & 8.20 \\
$0.10 \mathrm{~mol} / \mathrm{L}, \mathrm{NaHCO}_{3}, 5$ days & 4.76 & 8.10 \\
\hline
\end{tabular}

Fig. 1 shows the results of acidic functional group determination using the Boehm method. For both untreated and alkali-treated rice hull charcoal, acidic functional groups were detected following carbonization at $400{ }^{\circ} \mathrm{C}$. The amount of acidic functional groups on rice hull charcoal before and after alkali treatment decreased in the order of alkali treatment with $\mathrm{NaHCO}_{3}$ aqueous solution and alkali treatment with $\mathrm{NaOH}$ aqueous solution. By contrast, only a small amount of acidic functional groups was detected in the material carbonized at $600{ }^{\circ} \mathrm{C}$, and no acidic functional groups were detected after alkali treatment.

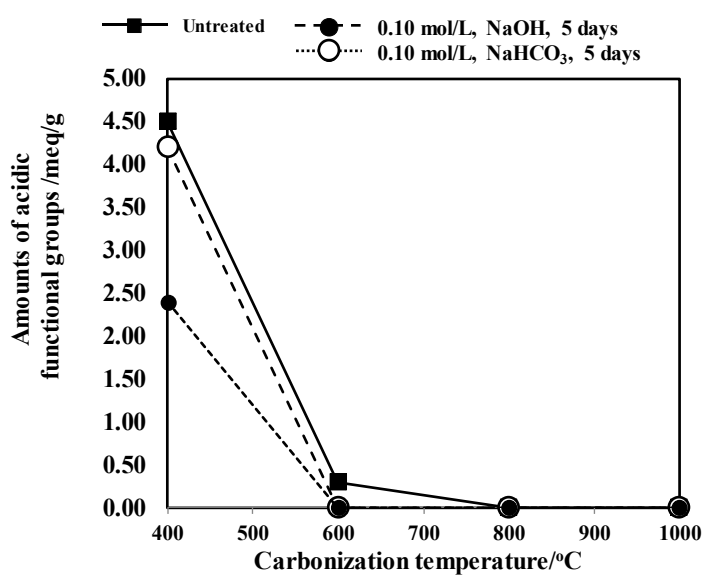

Fig. 1 Amount of acidic functional groups on the surface of rice hull charcoal.

Sodium is considered to be bound to the acidic functional groups of rice hull charcoal after alkali treatment. After alkali treatment, the chaff was washed, but it is thought that some bound sodium ions remained. Therefore, even if the rice hull charcoal was immersed in $\mathrm{NaOH}$ solution during acidic functional group determination using the Boehm method, some of the acidic functional groups were already bound to sodium ions, so were unavailable to bind to 
sodium ions in the solution. As a result, the amount of acidic functional groups after alkali treatment is likely higher than the measured value.
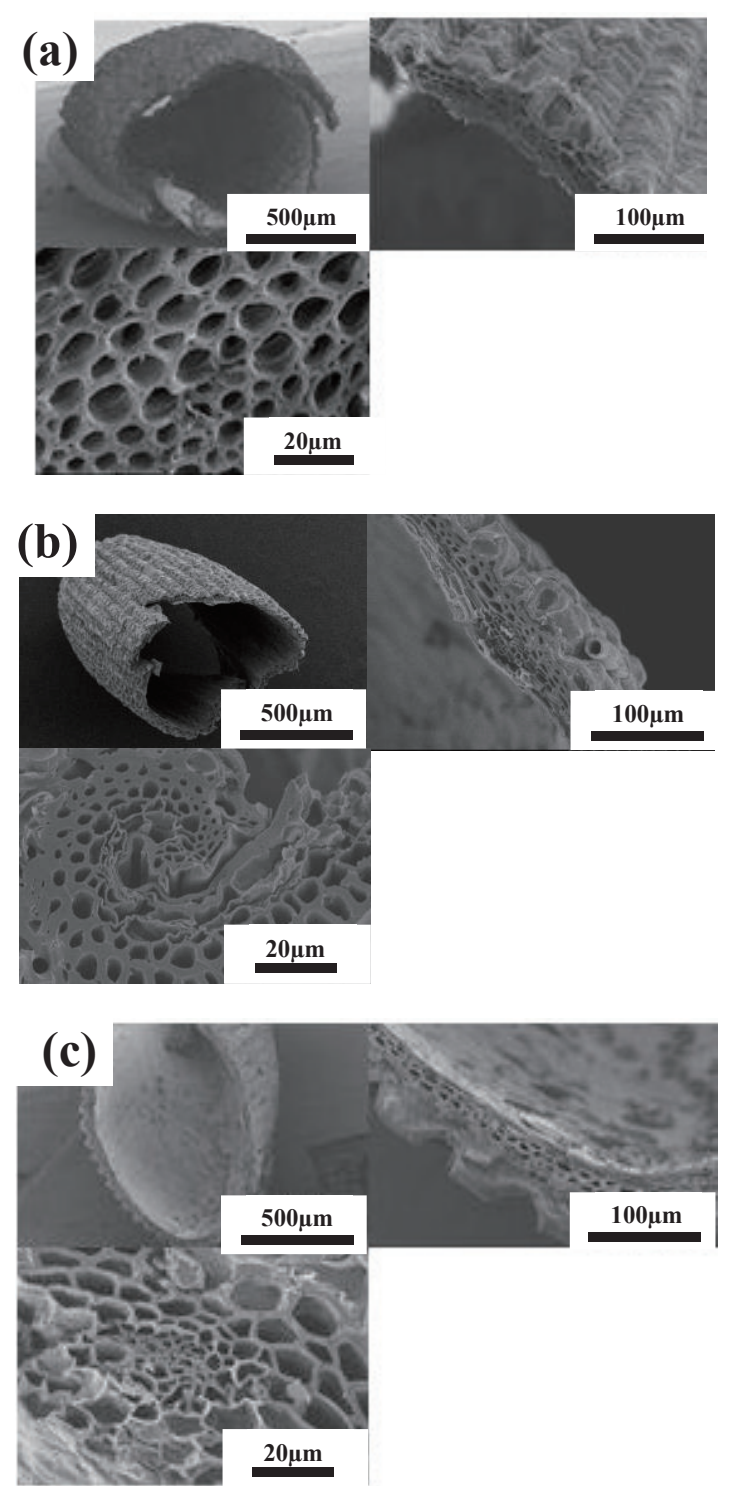

Fig. 2 SEM images of the surface of the rice hull charcoal samples carbonized at $400{ }^{\circ} \mathrm{C}$ after alkali treatment: (a) untreated, (b) $0.10 \mathrm{~mol} / \mathrm{L}, \mathrm{NaOH}, 5$ days, and (c) $0.10 \mathrm{~mol} / \mathrm{L}$, $\mathrm{NaHCO}_{3}, 5$ days .

The acid functional groups on rice hull charcoal carbonized above $800{ }^{\circ} \mathrm{C}$ may have burned during carbonization. As the amount of acidic functional groups such as carboxyl and phenolic hydroxyl groups decrease with heating above $580{ }^{\circ} \mathrm{C}$, it is considered that the acidic functional groups of rice hull charcoal carbonized at high temperature decreased or disappeared. Therefore, it is likely that acidic functional groups did not exist before and after alkali treatment.

Table I shows the specific surface area measurement results of rice hull charcoal. Even when rice hull charcoal was treated with alkali, the specific surface area did not increase, remaining around $5 \mathrm{~m}^{2} / \mathrm{g}$ or less.

Fig. 2 shows SEM images of each rice hull charcoal sample. The rice hull charcoal before and after alkali treatment has macroshell and microshell structures. In addition, porous parts are observed in the woody layer.

3.2 Pore size distribution measurement of rice hull charcoal

The pore size distribution measurement results of the rice hull charcoal before and after alkali treatment are shown in Fig. 3. In the case of alkali-treated rice hull charcoal, it appears that the volume of several nanometer sized pores is increased compared to that of the untreated sample.

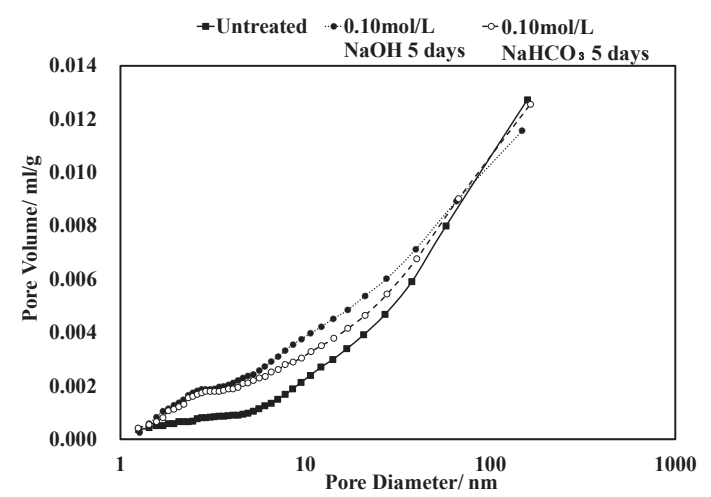

Fig. 3 Measurement of the pore distribution of rice hull charcoal carbonized at $400{ }^{\circ} \mathrm{C}$ before and after alkali treatment.

\subsection{FTIR measurement of rice hull charcoal}

The FTIR spectra of the rice hull charcoal before and after alkali treatment are shown in Fig. 4. In each spectrum, there is an absorption band near $1700 \mathrm{~cm}^{-1}$, which is characteristic of $\mathrm{C}=\mathrm{O}$ stretching. Furthermore, there is an absorption band near $3300 \mathrm{~cm}^{-1}$, most likely arising from $\mathrm{O}-\mathrm{H}$ stretching. The spectrum of the alkali-treated rice hull charcoal has a more intense $\mathrm{O}-\mathrm{H}$ band $\left(3300 \mathrm{~cm}^{-1}\right)$ than that of the untreated sample. Thus, alkali treatment increased the number of acidic O-H groups. In addition, it has been reported that lactone rings exist in activated carbon ${ }^{7)}$. Possibly, lactone rings are also present in the untreated rice hull charcoal used in this study. It has been reported that alkaline treatment cleaves the lactone ring of charcoal into a phenolic hydroxyl group and carboxyl acid group, as shown in Fig. 5, increasing the valence of the acidic functional groups ${ }^{8)}$.

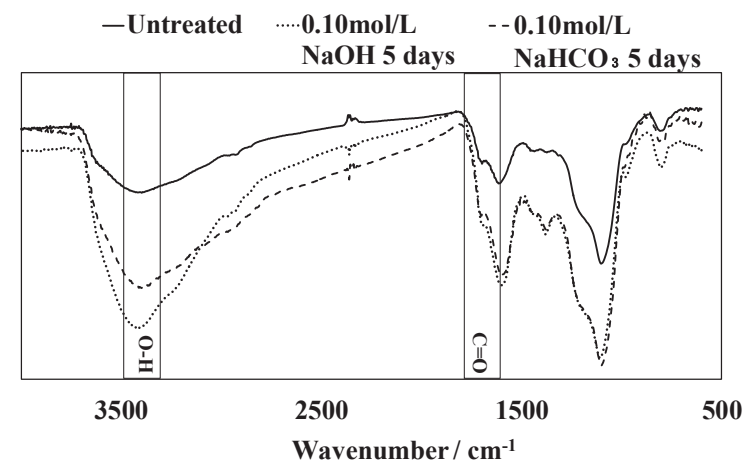

Fig. 4 Measurement of the pore distribution of rice hull charcoal carbonized at $400{ }^{\circ} \mathrm{C}$ before and after alkali treatment. 
<smiles>CCc1c(C(=O)O)ccc(O)c1-c1ccc(O)cc1C(=O)O</smiles>

Untreated

Alkali Treated

Fig.5 Schematic diagram of lactone ring of rice hull charcoal before and after alkaline treatment.

\subsection{Cesium/strontium adsorption experiments}

Fig. 6 shows the cesium adsorption of rice hull charcoal before and after alkali treatment. The amounts of adsorbed cesium in the rice hull charcoal carbonized at 400 and $600{ }^{\circ} \mathrm{C}$ was increased by alkali treatment. The amount of cesium adsorbed by the rice hull charcoal carbonized at $400{ }^{\circ} \mathrm{C}$ was increased by about 1.5 times by alkali treatment. By contrast, the amounts of cesium adsorbed by the rice hull charcoal carbonized at 800 and $1000{ }^{\circ} \mathrm{C}$ were slightly improved compared with the untreated sample when subjected to alkali treatment with $\mathrm{NaOH}$ aqueous solution. However, the amounts of cesium adsorbed by the rice hull charcoal treated with $\mathrm{NaHCO}_{3}$ aqueous solution were lower than that of the untreated sample. In addition, the adsorbed amount was about 4 times that of the natural zeolite measured for comparison.

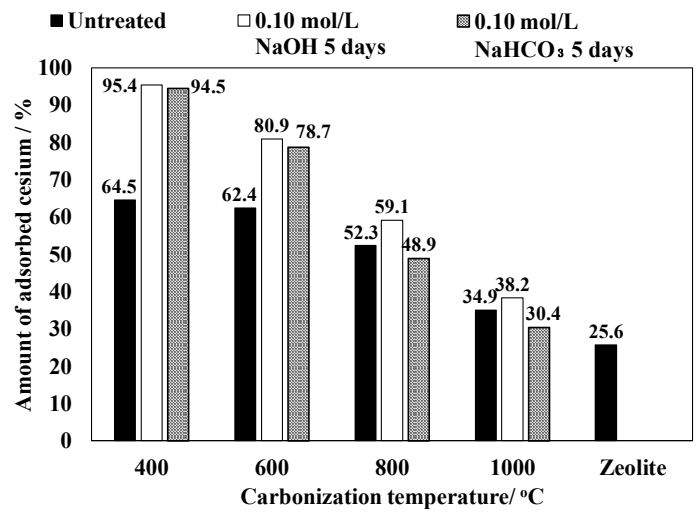

Fig. 6 Result of cesium adsorption of rice hull charcoal and natural zeolite carbonized at each temperature before and after alkali treatment.

The strontium adsorption of rice hull charcoal before and after alkali treatment is shown in Fig. 7. As with the cesium adsorption experiment results, the amount of strontium adsorbed by the rice hull charcoal carbonized at 400 and $600{ }^{\circ} \mathrm{C}$ was increased by alkali treatment. The amount of strontium adsorbed by the rice hull charcoal carbonized at $400{ }^{\circ} \mathrm{C}$ was increased about three times after alkali treatment, reaching $100 \%$. The amount of strontium adsorbed by rice hull charcoal carbonized at $600{ }^{\circ} \mathrm{C}$ increased after alkali treatment with $\mathrm{NaOH}$ aqueous solution. However, the amount of strontium adsorbed by rice hull charcoal treated with $\mathrm{NaHCO}_{3}$ aqueous solution decreased. By contrast, the amount of strontium adsorbed by rice hull charcoal carbonized at either 800 or $1000^{\circ} \mathrm{C}$ was lower for both alkali-treated samples than for the untreated sample. This value is about four times higher than the zeolite measured for comparison.

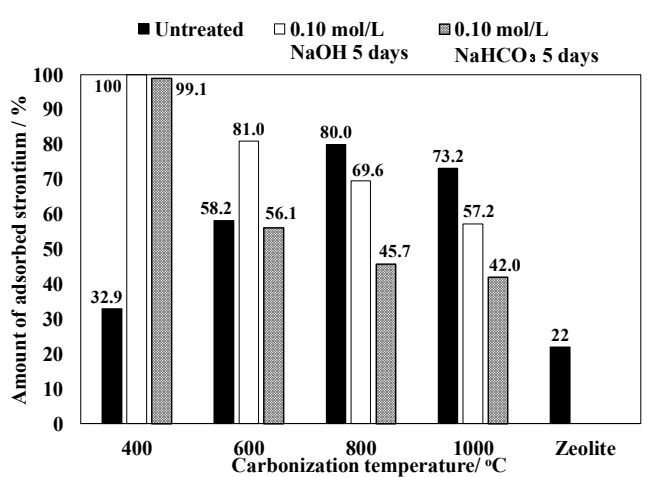

Fig. 7 Strontium adsorption of rice hull charcoal and natural zeolite carbonized at each temperature before and after alkali treatment.

As described above, it was found that the adsorption of cesium and strontium was improved by alkali treatment of rice hull charcoal carbonized at $400^{\circ} \mathrm{C}$.

The $\mathrm{pH}$ of a solution of a substance having acidic functional groups affects the adsorption ability of cesium and strontium ${ }^{9}$. When the $\mathrm{pH}$ is high, the negative charge of the substance increases, so that cesium and strontium are likely to be retained. In this study, the $\mathrm{pH}$ of rice hull charcoal was increased by treating rice hull charcoal with alkali; thus, as a result of the increase in negative charge, it is considered that the adsorption capacity was improved ${ }^{10)}$. Furthermore, it is considered that the adsorption ability of cesium and strontium was improved by increasing the valence of acidic functional groups by alkali treatment.

In addition, according to the pore distribution measurements, the mesopore volume of rice hull charcoal carbonized at $400{ }^{\circ} \mathrm{C}$ were slightly increased by the alkali treatment. According to Kobayashi et al. ${ }^{4)}$, there is a correlation between the mesopore volume and the cesium and strontium adsorption amounts. Furthermore, in this study, the increase in mesopores may have improved the adsorption amount after alkali treatment.

\subsection{Cesium/strontium desorption experiments}

The results of experiments for the desorption of cesium from the rice hull charcoal before and after alkali treatment are shown in Fig. 8. The alkali-treated rice hull charcoal showed less desorption than the untreated sample

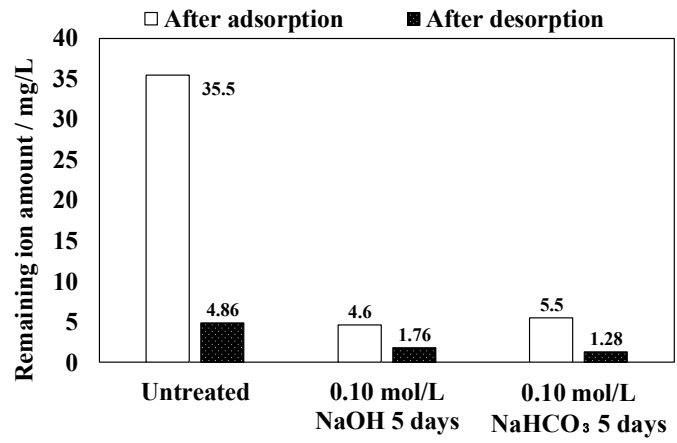


Fig. 8 Results of cesium desorption experiment of rice hull charcoal carbonized at $400{ }^{\circ} \mathrm{C}$ before and after alkali treatment.

The results of the strontium desorption experiments on rice hull charcoal before and after alkali treatment are shown in Fig. 9. Similarly with the cesium desorption experiments, the desorption amount of alkali-treated rice hull charcoal was less than that of untreated rice hull charcoal.

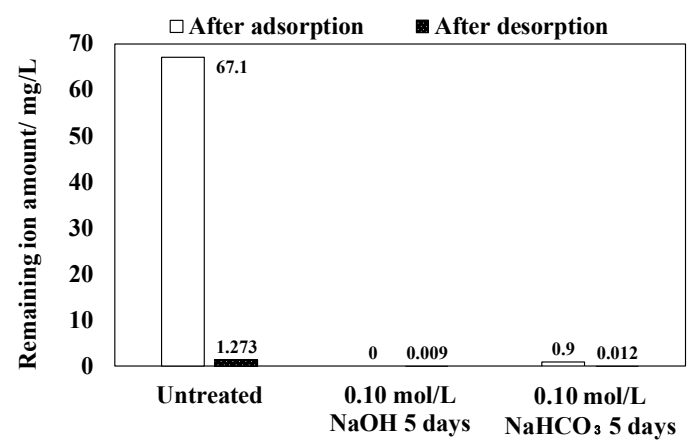

Fig. 9 Results of strontium desorption experiment of rice hull charcoal carbonized at $400{ }^{\circ} \mathrm{C}$ before and after alkali treatment.

In this study, the desorption of cesium and strontium was reduced by the alkali treatment. Therefore, it is considered that the amount of physical adsorption of cesium and strontium to the surface and pores of rice hull charcoal was not changed significantly. Generally, adsorption by chemisorption is stronger than physical adsorption ${ }^{5}$. For this reason, it is considered that the desorption amount decreased because of increased chemisorption in the alkalitreated rice hull charcoal.

\section{CONCLUSIONS}

The authors have investigated the relationship between the changes in the physical properties of rice hull charcoal after alkali treatment and its adsorption ability for cesium and strontium. The following points can be made.

1. The $\mathrm{pH}$ of rice hull charcoal increased after alkaline treatment.

2. The change in the specific surface area after alkali treatment was small. According to the pore distribution measurements, in the case of rice hull charcoal carbonized at $400{ }^{\circ} \mathrm{C}$, the mesopores were slightly increased by the alkali treatment. This increase may have contributed to the improved adsorption amounts of cesium and strontium.

3. The rice hull charcoal carbonized at $400{ }^{\circ} \mathrm{C}$ treated with alkali showed improved adsorption capacity for cesium and strontium and decreased desorption amount. The reason for this is assumed to be due to the following changes in the rice hull charcoal after alkaline treatment: (1) the $\mathrm{pH}$ increase and corresponding increase in negative charge, (2) the valence increase by cleavage of the lactone rings present on the surface, and (3) the mesopores increased.

As described above, the adsorption capacity of rice hull charcoal was improved by alkali treatment. Because rice hull charcoal is an abundant resource and its volume can be reduced by combustion, it could be used as a radiation absorbing material instead of zeolite, either in part or totally.

\section{REFERENCES}

[1] Mimura Hitoshi, Yamagishi Isao, and Akiba Kenichi, "Removal of radioactive cesium and strontium by zeolite," Chem. Soc. Jpn., 3, 621-627 (1989)

[2] Mitsumasa Kimata, Hiroyuki Imano, Naoya Kotake, and Masahiro Hasegawa, "Preparation of composite materials of rice husk powder and synthetic polymer by mechanochemical polymerization," J. Soc. Powder Technol. (Jpn.), 45, 484-490 (2008)

[3] Seiji Kumagai, Keiji Sasaki, Yoshie Shimizu, Koichi Takeda, "Adsorption of gaseous aldehydes on carbonized rice husk," J. Assoc. Mater. Eng. Res., 20, 34-38 (2007)

[4] Shinya Kobayashi, Kosuke Noda, Hirohumi Shibata, Soichiro Matsubara, Norihisa Kawamura, and Toru Nonami, "Adsorption properties of rice hull charcoal for cesium and strontium in aqueous solution," J. Soc. Mater. Sci.(Jpn.), 67, 898-903 (2018)

[5] Makoto Kobayashi, "Removal of radioactive Cs from soil by bio-char application," J. Jpn. Soc. Atmos. Environ, 46, 217-223, (2011)

[6] Katsuya Konno, Yuya Ohba, Kaoru Onoe, and Tatsuaki Yamaguchi, "Preparation of activated carbon having the structure derived from biomass by alkali activation with $\mathrm{NaOH}$, and its application for electric double-layer capacitor," TANSO, 231, 2-7, (2008)

[7] Sensho Honma and Toshimitsu Hata, "Immobilization of metallic ions on heat-treated wood from aqueous solution -Influences of alkali metal ions, temperature and pH," J. For. Prod. Res. Inst., 546, 38-40, (2018)

[8] Kazuya Yoshihara, Masami Aikawa, and Motoi Machida, "Influence of solution $\mathrm{pH}$ and background electrolytes on heavy metals ions adsorption by activated carbons from aqueous solutions," TANSO, 237, 61-66, (2009)

[9] Noriko Yamaguchi, Yusuke Takada, Kentarou Hayashi, Satoru Ishikawa, Masato Kuramata, Sadao Eguchi, Seiko Yoshikawa, Atsushi Sakaguchi, Kei Asada, Rota Wagai, Tomoyuki Makino, Ikuko Akahane and Shuntaro Hiradate, "Behavior of radio caesium in soil-plant systems and its controlling factor", Bulletin of the National Institute of AgroEnvironmental Sciences, 31，75-129, (2012

[10] Ikuo Abe, "Carbonization and activation techniques for production of carbonaceous adsorbent," TANSO, 211, 21-29, (2004)

(Received March 22, 2019; Accepted August 30, 2019; Published Online October 1, 2019) 